\title{
Massive Proteinuria which Rapidly Resolved after Discontinuation of Regorafenib
}

\author{
Mehmet Zahid Kocak, Gulali Aktas*, Satilmis Bilgin, Tuba Taslamacioglu Duman, Ozge Kurtkulagi, \\ Burcin Meryem Atak, Irem Emir Ozcil, Haluk Savli
}

Department of Internal Medicine, Abant Izzet Baysal University Hospital, Bolu, Turkey.

\begin{abstract}
Regorafenib is treatment option of cancers. It is associated with several adverse effects, including proteinuria. We report a massive proteinuria associated with regorafenib which resolved quickly after discontinuation of the drug. A 63 year old male presented with diarrhea and bilateral swallowing of legs. In urinanalysis, $18.5 \mathrm{~g}$ /day of proteinuria was detected. All symptoms, including proteinuria, resolved after discontinuation of regorafenib. Despite cessation of regorafenib treatment is advised in case of proteinuria, we suggest that regorafenib associated proteinuria could be rapidly resolved after discontinuation of the drug.
\end{abstract}

Keywords: Regorafenib, Massive proteinuria, Treatment, Cancer, Adverse effect, Diarrhea.

\section{INTRODUCTION}

Regorafenib is a novel multikinase inhibitor that targets vascular endothelial growth factor receptor 1-3 (VEGFR), platelet derived growth factor receptor (PDGFR) beta and inhibitors of oncogenic receptor tyrosine kinases such as, CD117 (c-KIT), RET and RAF [1]. Main cancers that regorafenib commonly used includes hepatocellular carcinoma, metastatic colorectal carcinoma and gastrointestinal stromal tumors [2]. Although it provides an effective treatment in these patients, regorafenib related side effects involving several organ systems have been reported. These side effects include hypertension, neurological (headache, voice disturbance), dermatological (palmar-plantar erythrodysesthaesia, skin rash, alopecia), metabolic (hypophosphatemia, hypocalcaemia, hypokalaemia, hypernatremia, hypothyroidism, weight loss), gastrointestinal (elevated serum amylase and lipase levels, anorexia, mucositis, nausea and vomiting), hepatic (hyperbilirubinaemia, elevated transaminases), and hematologic (anaemia, lymphopaenia, thrombocytopenia, neutropenia, haemorrhage, increased INR) signs and symptoms [3].

Proteinuria is not a rare side effect of regorafenib and reported about $33-84 \%$ of the subjects received regorafenib based regimen. However, most of the cases suffer from mild proteinuria (grade 1 and 2) and severe proteinuria related with regorafenib is very rare $(<1 \%)$.

In present study, we aimed to report a patient with metastatic hepatocellular carcinoma that was on regorafenib treatment whom developed nephrotic level massive proteinuria $(>20 \mathrm{~g} /-$ day) and rapidly ameliorated after discontinuation of regorafenib.

*Address correspondence to this author at the Department of Internal Medicine, Abant Izzet Baysal University Hospital, Golkoy, 14280, Bolu, Turkey. Email: draliaktas@yahoo.com

\section{CASE REPORT}

A 63 year old male showed up in our clinic complaining of diarrhoea and bilateral swallowing of legs. Leg swallowing and diarrhoea was developed 1 month ago and gradually increased. Anorexia, fatigue, nausea, shivering and fever have been occurred 4 days before presentation. He stated formless faeces diarrhoea 8 to 10 times a day. He did not have any complaints of vomiting, cough, sputum production or dysuria.

Swallowing of his legs has worsen in time since it began 30 days ago. Swallowing leaves fingerprint after applying pressure on by finger. Moxifloxacin 500mg daily was prescribed for a diagnosis of pneumonia 3 days ago in another health institution that he previously showed up. He presented to our outpatient clinics because his complaints did not resolved.

His medical history was positive for chronic hepatitis B for 20 years. Three years ago, a diagnosis of hepatocellular carcinoma (HCC) was established in another institution where he received radionuclide therapy as a first line treatment for HCC. He has been prescribed sorafenib $400 \mathrm{mg}$ twice a day, since the first line treatment was ineffective. Sorafenib was switched to regorafenib $160 \mathrm{mg}$ daily, after 2 years because of heart failure developed (ejection fraction of left ventricle was $30 \%$ ), which thought to be associated with sorafenib. He was also started on ramipril, spironolactone and carvedilol for the treatment of heart failure.

Since palmar and plantar desquamation occurred in second month of the regorafenib treatment, the dosage of regorafenib was reduced to $80 \mathrm{mg} /$ day and he received that daily dose for 2 months until he presented to our clinic. His family history was unremarkable.

On physical examination, he was ill appeared, was conscious, oriented and cooperated. Vital signs were as follows: blood pressure was $130 \mathrm{mmHg}$ systolic and $80 \mathrm{mmHg}$ diastolic, 
heart rate was 72 /minute, body temperature was 36,5 Celsius degree, and respiratory rate was $22 /$ minute. Lung sounds were decreased bilaterally at the bottom of the chest and tuber sufl was audible on the bottom of the right side. There were no crackles or rhonchi. The abdomen was distended and minimal ascites was detected. Bilateral $4+$ pretibial oedema which had left fingerprint was noted. Rest of the findings in physical examination were normal.

Initial blood tests were as follows: white blood cell count: 11300/mm3, neutrophil count: 8600/mm3, haemoglobin: 12.9 $\mathrm{g} / \mathrm{dL}$, platelet count: $130000 / \mathrm{mm} 3$, blood urea: $143 \mathrm{mg} / \mathrm{dL}$, serum creatinine: $1.81 \mathrm{mg} / \mathrm{dL}$ (glomerular filtration rate: 37 $\mathrm{mL} /$ minute), albumin: $2.2 \mathrm{~g} / \mathrm{dL}$, total protein $4.3 \mathrm{~g} / \mathrm{dL}$, c-reactive protein: $84 \mathrm{mg} / \mathrm{L}$, INR:1.76, aspartate transaminase: 69 $\mathrm{U} / \mathrm{L}$, alanine transaminase: $42 \mathrm{U} / \mathrm{L}$, gamma glutamyl transferase: $277 \mathrm{U} / \mathrm{L}$, alkaline phosphatase: $418 \mathrm{U} / \mathrm{L}$, total bilirubin: $0.73 \mathrm{mg} / \mathrm{dL}$, direct bilirubin: $0.2 \mathrm{mg} / \mathrm{dL}$, lactate dehydrogenase: $342 \mathrm{U} / \mathrm{L}$.

Urinalysis was remarkable for $3+$ blood and $3+$ protein. Microscopic evaluation of faeces was unremarkable. Venous blood gas analysis revealed blood $\mathrm{pH}$ : 7.25, pCO2: 22.4mmHg, pO2: $39 \mathrm{mmHg} \mathrm{HCO} 3: 9.5 \mathrm{mmol} / \mathrm{L}$ and lactate: $1.38 \mathrm{mmol} / \mathrm{L}$. Negative urinary anion gap suggested the metabolic acidosis was due to gastrointestinal loss.

Chest radiography was remarkable for bilateral multiple metastatic nodular lesions and suspicious consolidation in basal segments of right lung.

In abdominal sonography, liver parenchyma was heterogeneous and multiple hypo-echoic focal solid masses (greatest one is located in segment 8 with a diameter of $17 \times 19 \mathrm{~mm}$ ) which were consisted of HCC were noted. Other sonographic findings include minimal ascites.

Cardiac echocardiography revealed a left ventricle ejection fraction of $55 \%$ which means significant improvement since the time of cessation of sorafenib.

The patient's laboratory tests prior to regorafenib treatment revealed normal urinalysis and a serum creatinine at 0.7 $\mathrm{mg} / \mathrm{dL}$, thus, the patient was hospitalized with the diagnoses of acute kidney injury and pneumonia.

On admission to ward, moxifloxacin $400 \mathrm{mg} /$ day and Tenofovir $245 \mathrm{mg}$ /day treatments are continued after obtaining blood, urine, sputum and fecal cultures. Ramipril, carvedilol and spironolactone were discontinued. An analysis of 24 hours of urine revealed $18.5 \mathrm{~g}$ proteinuria. Regorafenib treatment was discontinued at once. Blood cultures were positive for Escherichia coli, which was resistant to moxifloxacin. Therefore, ertapenem $1 \mathrm{~g} /$ day was switched instead of moxifloxacin.

Metabolic acidosis, which is improper with creatinine clearance was thought to be a consequence of long lasting diarrhoea and $500 \mathrm{mg}$ of Na-bicarbonate administered three times daily. Rifaximin was also initiated for diarrhoea after consultation with a gastroenterologist. Loperamide $2 \mathrm{mg}$ twice daily improved the frequency of diarrhoea. Metabolic acidosis was also completely resolved (pH: 7.43, pCO2: $34 \mathrm{mmHg}$, HCO3: $23 \mathrm{mmol} / \mathrm{L}$ ).

Since oral nutrition was insufficient, parenteral nutrition solution infused daily. Albumin solution was also administered. Treatment with furosemide $20 \mathrm{mg}$ twice a day was initiated for leg swallowing.

Pretibial oedema of the patient resolved during clinical follow-up. Urinary protein excretion reduced to $4.942 \mathrm{~g} /$ day and serum creatinine to $1.3 \mathrm{mg} / \mathrm{dL}$ at $7^{\text {th }}$ day after discontinuation of regorafenib. Therefore, ramipril resumed at a dose of $2.5 \mathrm{mg}$ daily.

The patient was developed restlessness, sleep disturbance, delirium and hallucination at $5^{\text {th }}$ day of ertapenem treatment. Haloperidol $1 \mathrm{mg}$ twice and quetiapine $50 \mathrm{mg}$ once daily ordered after consultation with neuropsychiatry department. A cranial magnetic resonance imaging study ruled out intracranial events or metastatic lesions. Ertapenem was accused for delirium and discontinued at $11^{\text {th }}$ day, then, delirium gradually resolved in a couple of days. Both quetiapine and haloperidol were discontinued.

General performance of the patient improved and pretibial oedema completely resolved at $16^{\text {th }}$ day of the hospitalization. His appetite got well and oral nutrition of the patient was improved. At $30^{\text {th }}$ day, proteinuria was as low as $3.96 \mathrm{~g} /$ day. He discharged with recommendation of furosemide $40 \mathrm{mg}$ every other day and control visit with in a month. At first clinic control visit $15^{\text {th }}$ day after discharge $\left(45^{\text {th }}\right.$ day after cessation of regorafenib), the paint was well and proteinuria was reduced to $268 \mathrm{mg} /$ day. Change in the level of proteinuria during and after regorafenib treatment was summarized in Fig. (1).

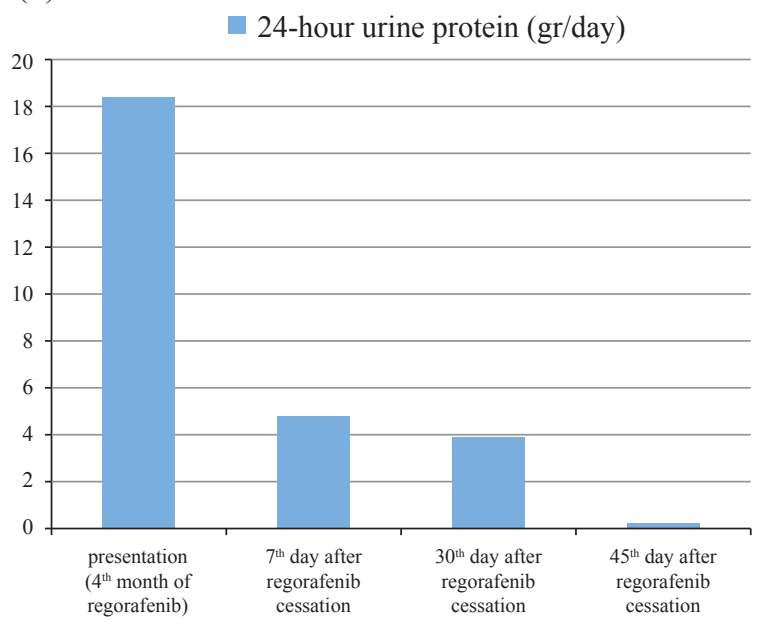

Fig. (1). Change in the Level of Proteinuria During and after Regorafenib Treatment. 


\section{DISCUSSION}

We presented a severe proteinuria related with regorafenib that resolved after cessation of treatment. To the best of our knowledge, this is the first case in literature that reported rapidly resolving of massive proteinuria due to regorafenib.

Proteinuria is graded as follows; grade I: less than $1 \mathrm{~g}$ /day proteinuria, grade II: 1 to $3.5 \mathrm{~g}$ /day proteinuria and grade III: $\geq 3.5 \mathrm{~g} /$ day proteinuria [4]. The proteinuria in patient that we present could be classified as grade III, according to Common Terminology Criteria for Adverse Events criteria.

Regorafenib may have side effects in multiple organs and systems, including proteinuria. In 2013, proteinuria was reported in $7 \%$ of patients received regorafenib treatment [5]. In other studies, proteinuria of any grade was reported as $11 \%$ [6] and 47\% [7]. Regorafenib associated severe proteinuria (grade III) has been reported as less as $1 \%$ in Grothey et al.'s study [5]. However, two other reports in literature notified proteinuria as high as 3\% [6], and 7\% [7] in patients received regorafenib treatment. Proteinuria in the patient here we present was grade III and could be defined as severe or massive with $18.5 \mathrm{~g}$ daily protein loss in urine.

Regorafenib associated proteinuria is more common in certain ancestry. In a study by Yoshino et al., any grade of proteinuria in Japanese and non-Japanese population were reported as $40 \%$ and $2 \%$, and grade 3 proteinuria was reported as $6 \%$ and less than $1 \%$, respectively [8]. The patient we reported here was Caucasian ancestry.

If the degree of proteinuria in a patient receiving regorafenib treatment is greater than $2 \mathrm{~g} / \mathrm{day}$, holding of regorafenib is advised until proteinuria reduces to $<2 \mathrm{~g} /$ day [3]. If proteinuria is at nephrotic level (grade III), discontinuation of regorafenib is recommended [3]. Since the urinary protein excretion of present case was $18.5 \mathrm{~g} /$ day, we promptly discontinued the regorafenib.

Regorafenib has a variety of side effects. These are mainly related to dermatological, gastrointestinal, cardiac and metabolic undesired effects. Of those regorafenib related side effects, such as diarrhoea, anorexia and nausea were present in our case. All of these symptoms were resolved after cessation of the regorafenib treatment.

Underlying mechanism of proteinuria during regorafenib treatment is unclear, however, several mechanisms of regorafenib related proteinuria have been suggested, such as, immune-complex glomerulonephritis, thrombotic microangiopathy, cryoglobulinemia $[9,10]$. The exact mechanism of the proteinuria in present case is still unclear since renal biopsy was not needed due to rapid resolving of proteinuria.

In conclusion, we suggest that regorafenib associated proteinuria, even grade III, can be reversed rapidly after discontinuation of the drug. Physicians should aware of this side effect and spare enough time to follow up proteinuria after cessation of regorafenib. Further studies are needed whether regorafenib could be re-initiated in patients that benefit from treatment and with severe proteinuria that resolved quickly by only discontinuation of this medicine.

\section{AUTHORS' CONTRIBUTION}

All authors have contributed equally.

\section{CONFLICT OF INTEREST}

Declared none.

\section{ACKNOWLEDGEMENTS}

Declared none.

\section{REFERENCES}

[1] Ettrich TJ, Seufferlein T. Regorafenib. Recent Results Cancer Res 2014; 201: 185-96. DOI: 10.1007/978-3-642-54490-3_10

[2] Crona DJ, Keisler MD, Walko CM. Regorafenib: A novel multitargeted tyrosine kinase inhibitor for colorectal cancer and gastrointestinal stromal tumors. Ann Pharmacother 2013; 47(12): 1685-96. DOI: 10.1177/1060028013509792

[3] Krishnamoorthy SK, Relias V, Sebastian S, Jayaraman V, Saif MW. Management of regorafenib-related toxicities: A review. Therap Adv Gastroenterol 2015; 8(5): 285-97. DOI: $10.1177 / 1756283 X 15580743$

[4] US Department of Health and Human Services, National Institutes of Health, National Cancer Institute. Common Terminology Criteria for Adverse Events (CTCAE). 2017; Available at https://ctep.cancer.gov/protocoldevelop$\mathrm{m}$ e $\mathrm{nt} / \mathrm{e} 1$ e c t r o n i c - a p p 1 i cations/docs/CTCAE_v5_Quick_Reference_8.5x11.pdf.)

[5] Grothey A, Van Cutsem E, Sobrero A, et al. Regorafenib monotherapy for previously treated metastatic colorectal cancer (CORRECT): An international, multicentre, randomised, placebo-controlled, phase 3 trial. Lancet 2013; 381(9863): 303-12. DOI: 10.1016/S0140-6736(12)61900-X

[6] Bolondi L, Tak W, Gasbarrini A, et al. 6576 POSTER phase II safety study of the oral multikinase inhibitor regorafenib (BAY 73-4506) as second-line therapy in patients with hepatocellular carcinoma. Eur J Cancer 2011; 47: S464. DOI: 10.1016/S0959-8049(11)71887-1

[7] Furuse J, Sasaki Y, Okusaka T, et al. 1236 POSTER phase I study to assess the safety, tolerability and pharmacokinetics of the multikinase inhibitor regorafenib (BAY 73-4506) in Japanese patients with advanced solid tumours. Eur J Cancer 2011; 47: S155. DOI: 10.1016/S0959-8049(11)70848-6

[8] Yoshino T, Komatsu Y, Yamada Y, et al. Randomized phase III trial of regorafenib in metastatic colorectal cancer: Analysis of the CORRECT Japanese and non-Japanese subpopulations. Invest New Drugs 2015; 33(3): 740-50.

DOI: $10.1007 / \mathrm{s} 10637-014-0154-\mathrm{x}$

[9] Izzedine H, Massard C, Spano JP, Goldwasser F, Khayat D, 
Soria JC. VEGF signalling inhibition-induced proteinuria: Mechanisms, significance and management. Eur J Cancer 2010; 46(2): 439-48. DOI: 10.1016/j.ejca.2009.11.001
[10] Eremina V, Jefferson JA, Kowalewska J, et al. VEGF inhibition and renal thrombotic microangiopathy. N Engl J Med 2008; 358(11): 1129-36. DOI: 10.1056/NEJMoa0707330 